\title{
TAPPING OF LOST VELOCITY OF AIR FOR WIND MILL WITH SECOND ROTOR SET -UP
}

\author{
M. RAJNIKANTH \& M. SANTHOSH KUMAR \\ Department of Mechanical Engineering, Narasu's Sarathy Institute of Technology,
}

Anna University, Salem, Tamil Nadu, India

\begin{abstract}
Wind power is more efficient and affordable than it has ever been which has helped drive its popularity. It is important to understand that greater use of nation's abundant wind resources for electric power generation will help stabilize energy costs, enhance energy security, and improve our environment. Also, wind energy has been of prime importance as wind turbines can be used as stand-alone applications, or they can be connected to a utility power grid or even combined with a photovoltaic (solar cell) system. For utility-scale sources of wind energy, a large number of wind turbines are usually built close together to form a wind plant. Several electricity providers today use wind plants to supply power to their customers. Recent studies have highlighted the negative impacts of its use of environment and life especially on birds. But there should be a trade-off between its use and its impact.

This experimented study deals with maximization of wind energy by incorporating another wind rotor set-up adjacent to the main wind rotor so that the velocity of air that bypasses the main blades strikes the second rotor set -up thereby minimizing the percentage of bypassed air. An attempt is made to combine the outputs of these two and feed this output to turbine as a single input. By this method, the area required for installation can be reduced, at the same time maximize the conversion rate of wind into energy (power output). The objective is to increase the performance and reliability of next-generation wind technologies while lowering the cost of wind energy.
\end{abstract}

KEYWORDS: Velocity of Air \& Second Rotor Setup

Received: Jul 16, 2017; Accepted: Aug 09, 2017; Published: Aug 24, 2017; Paper Id: IJMPERDOCT20173

\section{INTRODUCTION}

Innovation in the design and manufacturing of wind power generation components continues to be critical to achieving our national renewable energy goals. To ensure the effective and greater use of wind energy, the technology must continue to evolve, building on earlier successes to further improve reliability of next-generation wind technologies, increase capacity factors, and reduce costs. While a previous focus on the industry was increasing the total nameplate capacity of wind turbines, the focus has shifted to the capacity factor of the turbine, which helps keeps energy cost low by providing the most possible power. Improved capacity factors and reduced cost of energy has enabled to go into more and more locations where the wind is lower. Development of longer, lighter rotor blades, taller towers, more reliable drive trains, and performance-optimizing control systems are the need of the hour.

Wind energy has been long time option for production of power which is one among the non conventional source of energy. This is so because, the wind comes from atmospheric changes, i.e. changes in temperature and pressure, thereby making the air move around the surface of the earth, all of which triggered by 
the sun. Humans have put these energy sources into use to achieve various purposes. In the early ages of its use, people constructed wind mills to generate energy meant for grinding grains. They also constructed mechanical wind pumps to pump large amounts of water into the cultivated fields.

Wind turbine captures the wind to produce energy using a simple setup consisting of a rotor which spins due to wind and the movement of the blades spinning is converted into power by the generator. Therefore, it is a non-polluting renewable resource of energy that creates power without using fossil fuels, without producing greenhouse effect or radioactive or toxic waste. Wind energy is non-conventional and renewable, clean energy. Power is generated when air passes through the turbine blades whose mechanical output is converted into electrical energy with the help of a generator. Wind turbines, like windmills, are mounted on a tower to capture most of the energy. At 100 feet (30 meters) or more above ground, they can take advantage of the faster and less turbulent wind. Turbines catch the wind's energy with their propeller-like blades. Usually, two or three blades are mounted on a shaft to form a rotor. A blade acts much like an airplane wing. When the wind blows, a pocket of low-pressure air forms on the downwind side of the blade. The lowpressure air pocket, then pulls the blade toward it, causing the rotor to turn. This is called lift. The force of the lift is actually much stronger than the wind's force against the front side of the blade, which is called drag. The combination of lift and drag causes the rotor to spin like a propeller, and the turning shaft spins a generator to make electricity. Wind power does not produce the greenhouse effect during operation and with the proposed set-up as mentioned earlier the utilization of land for installation is also reduced further.

Reports suggest that there is considerable increase in the use of wind energy as compared to fossil fueled energy making it clear the importance of this form of energy for the future. The installed wind power capacity of china has increased from 2599MW in 2006 to 145,104 MW in 2015, for the European Union it has increased from 48,122MW to 141,579MW between 2006 to 2015, for the United States it has increased from 11,603MW to 74,472 MW between 2006 to 2015. For India statistics show the increase as $76,270 \mathrm{MW}$ in $25,088 \mathrm{MW}$ for the same period ${ }^{1}$. This shows a lot has to be done for proper production methods and its utilization in our country.

The airflow at the blades is not the same as the air flow far away from the turbine. The very nature of the way in which energy is extracted from the air causes air to be deflected by the turbine. In the convectional turbine, the efficiency of the turbine is minimum compared to this turbine set-up since in this set-up the turbine has two rotors with blades to absorb the total velocity of the air. Also, in the convectional type only the $50 \%$ of the air velocity is absorbed, which can be termed as the useful velocity of air that is converted into electric power, including taking into consideration the conversion loss.

\section{WORKING PRINCIPLE}

Wind is simply defined as moving air. When the earth heats up from the sun rays it releases wind, this is a balanced reaction to cool the earth. The sun's heat is felt more on dry land than on the sea. The air has the tendency to expand and it reaches maximum high altitudes due to which cool air drops down and moves as the wind. Wind energy is generated by converting kinetic energy through friction process into useful forms such as mechanical energy and electricity.

In conventional wind turbines, there is a loss of $50 \%$ of air flow which triggered an attempt to maximize the utilization of the air flow such that the velocity of air that by-pass the first wind rotor is further absorbed by the second 
wind rotor attached at the back of the first wind rotor so that, the amount of air that bypasses it, is being considerably reduced. The outputs of these two rotors are coupled thereby reducing the conversion losses also since the output of the two turbines is combined mechanical losses would also be reduced.

\section{WIND SPEED CALCULATION}

The wind resource at a particular location is characterized by two measures, first one is wind speed and the second is wind direction. Using these two measures, the feasibility of installing a wind turbine can be determined. The average wind speed should be above $5 \mathrm{~m} / \mathrm{s}$ (18km per hour) so that, the installation of wind turbine is worthwhile. Ideal locations are generally away from the built-up areas like farms or on the coast. This is because, the more the buildings the less wind for the turbines. Setting up of wind turbine incurs huge capital, but it can be done in a very cost effective way of generating power. The denser the air, the more energy received by the turbine. Air density varies with elevation and temperature. Air is less dense at higher elevations than at sea level, and warm air is less dense than cold air. The turbines will produce more power at lower elevations and in locations with cooler average temperatures.

The amount of energy in the wind varies with the cube of the wind speed, for example, if the wind speed doubles; there is eight times more energy in the wind. Small changes in wind speed have a large impact on the amount of power available in the wind.

Approximate Velocity of wind stream after passing through the wind turbine.

If a stream of wind with density ( $\rho$ ) blows with a velocity (V) $\mathrm{m} / \mathrm{s}$ through an area of A sq. $\mathrm{m}$, then, the power of wind stream ${ }^{2}$ is given by:

$$
P_{w s}=1 / 2 \rho A V^{3}
$$

The theoretical maximum power co-efficient (efficiency) of a wind stream is 0.5926 .

However, most of the practical wind turbines run with power co-efficient (efficiency) at the range of $40 \%$. If, $10 \%$ of input energy is taken as a loss, then after passing through the wind turbine the power of the air becomes about $50 \%$ of the incoming stream.

Let

$\mathrm{V}_{\mathrm{i}}=$ velocity of incoming stream,

$\rho_{\mathrm{i}}=$ density of air,

$\mathrm{A}_{\mathrm{i}}=$ area through which the incoming air passes,

And

$V_{o} \rho_{o}$ and $A_{o}$ are the same for outgoing stream of air after extraction of energy from it,

Then

$1 / 2 \rho_{\mathrm{o}} \mathrm{A}_{\mathrm{o}} \mathrm{V}_{\mathrm{o}}^{3}=0.5 \cdot 1 / 2 \rho_{\mathrm{i}} \mathrm{A}_{\mathrm{i}} \mathrm{V}_{\mathrm{i}}^{3}(2)$

The applying continuity equation between the inlet and outlet of the turbine with the assumption that the density of the air does not change considerably, 
$A_{o} V_{o}=A_{i} V_{i}$

Or, $\mathrm{A}_{\mathrm{o}}=\left(\mathrm{A}_{\mathrm{i}} \mathrm{V}_{\mathrm{i}}\right) / \mathrm{V}_{\mathrm{o}}$

Putting this in Equation. (2) we get,

$\mathrm{V}_{\mathrm{o}}=0.707 \mathrm{~V}_{\mathrm{i}}$

Therefore, if we use a wind turbine in a field of air velocity of $10 \mathrm{~m} / \mathrm{s}$, after passing through wind turbine the velocity of air becomes $7 \mathrm{~m} / \mathrm{s}$.

Theoretically, only $30 \%$ of air flow is utilized for conversion. To avoid loss of this volume a design is developed which is shown in Figures and are illustrated.

\section{DESIGN OF BLADE}

In wind turbine blades, blades could be very thin at the end, and there's also a little twist to it, so maintaining fluid dynamic contact on the blade across of range of wind velocities and different angles of attack and different yaw is a complex fluid mechanics problem, The blades are affected by two different forces: torque, which turns the blades and creates energy, and thrust, which pushes against the turbine. Dealing with thrust can be difficult when designing blades. The blade is curved at the tip, which, unlike the vast majority of blades in use, is designed to take maximum advantage of all wind speeds, including slower wind speeds. For the experiment, the blades are made of PVC pipes.

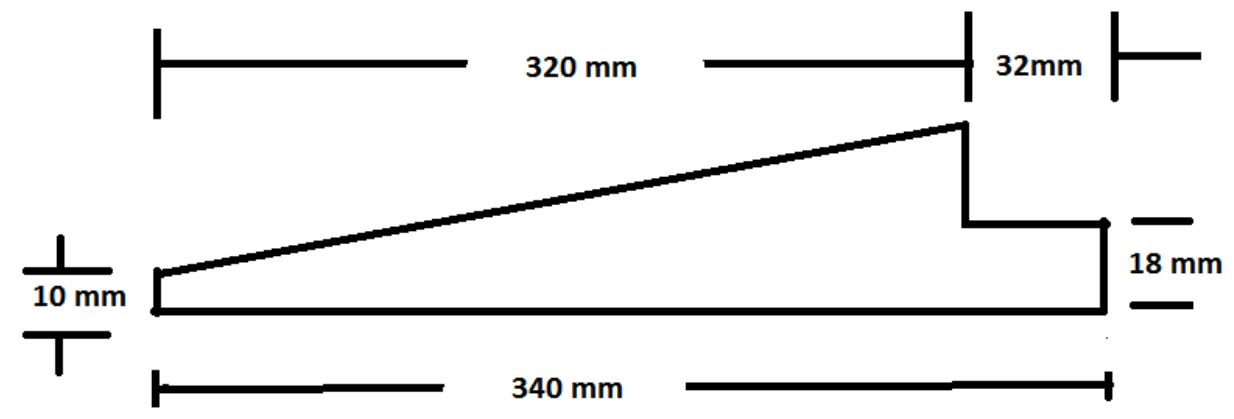

Figure 1: Dimension of the blade

The figure shows dimension of the blade design. The total length of the experimental blade design is $340 \mathrm{~mm}$ out of which $320 \mathrm{~mm}$ is the tapered length and the other $20 \mathrm{~mm}$ is used for voting purposes whose width would be $19 \mathrm{~mm}$. One end of the taper has $10 \mathrm{~mm}$ and the other is about $50 \mathrm{~mm}$. 

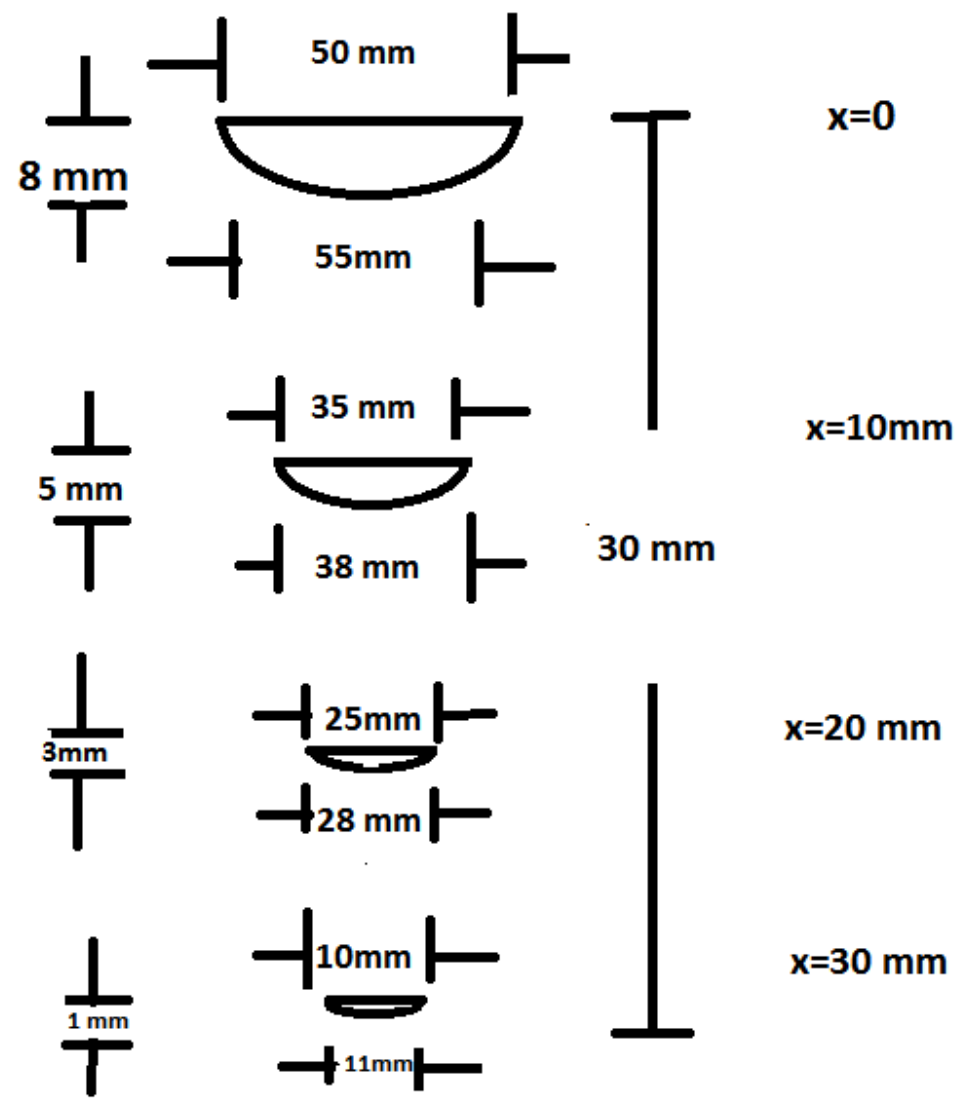

Figure 2: Dimension of Curved Section of Blade at Various Lengths

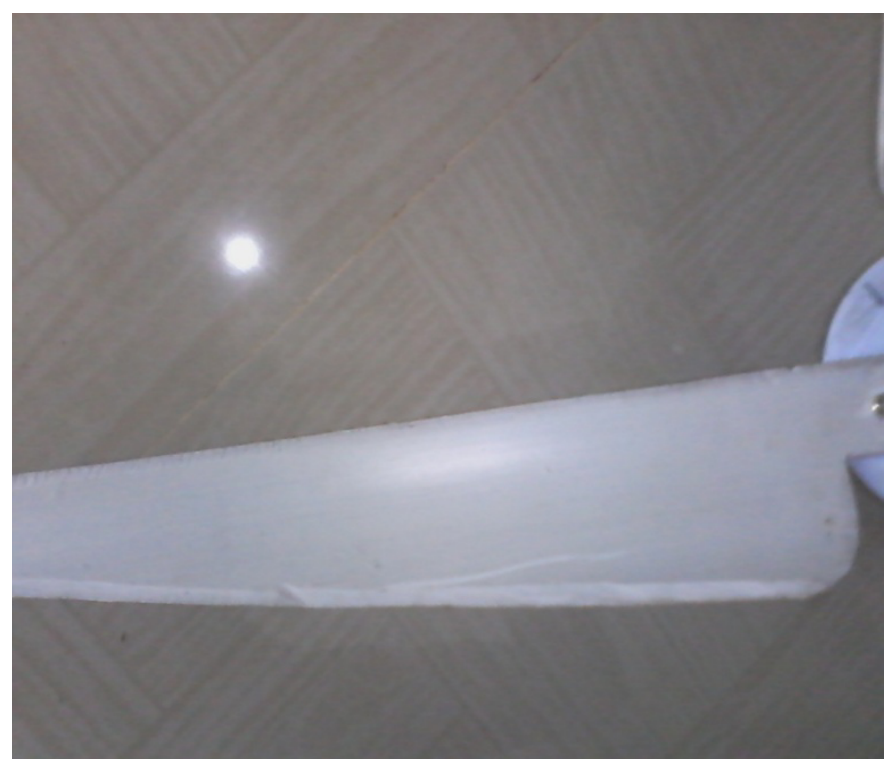

Figure 3: Three Dimensional View of the Blade

The figure shows three dimensional view of the blade. The blade is made up of PVC pipes for experiment. 


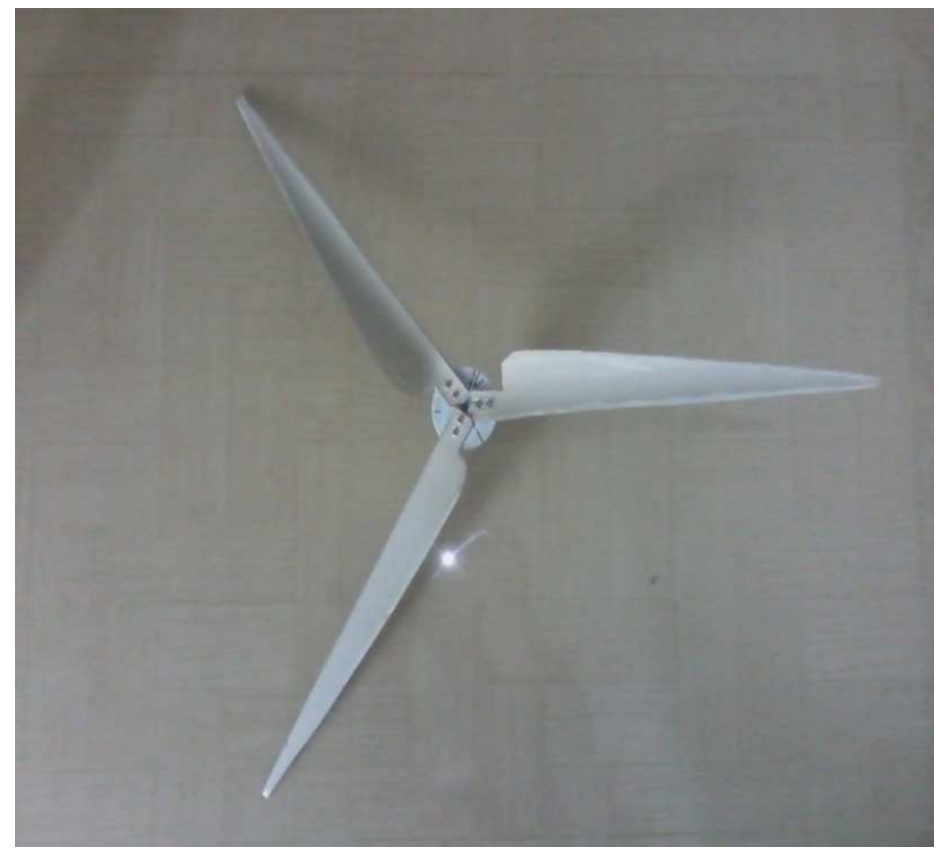

Figure 4: Three Dimensional View of the Blade Set-Up

The figure shows three dimensional view of the blade set-up in which three blades are bolted to rotor. For tightening and fixing purposes ordinary screws were used.

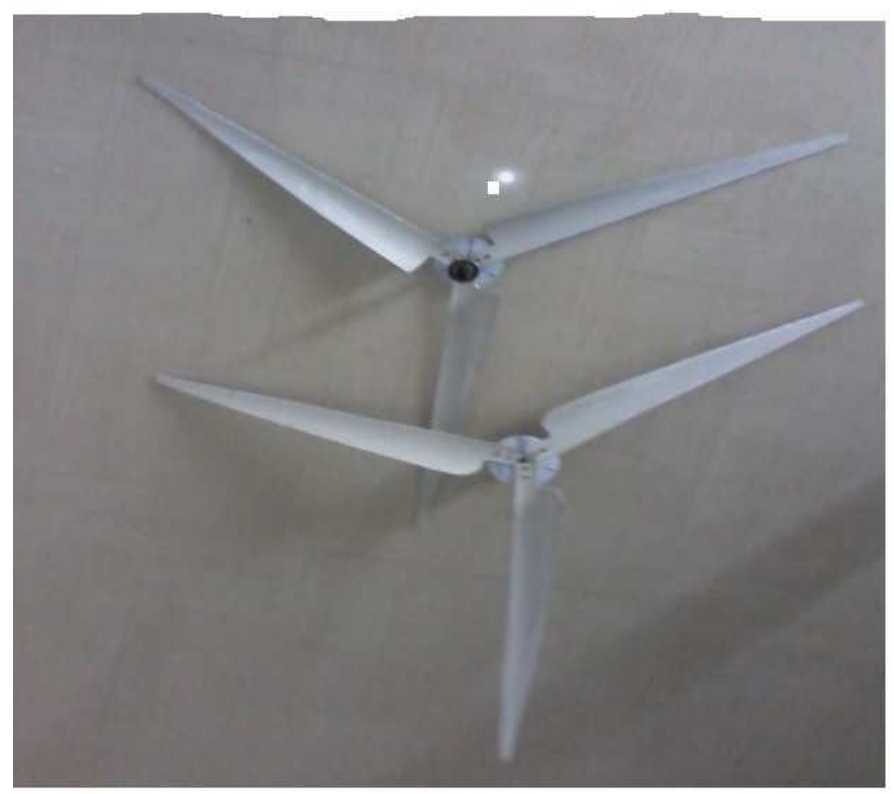

Figure 5: Two Set of Blades for Front and Back Set-Up 


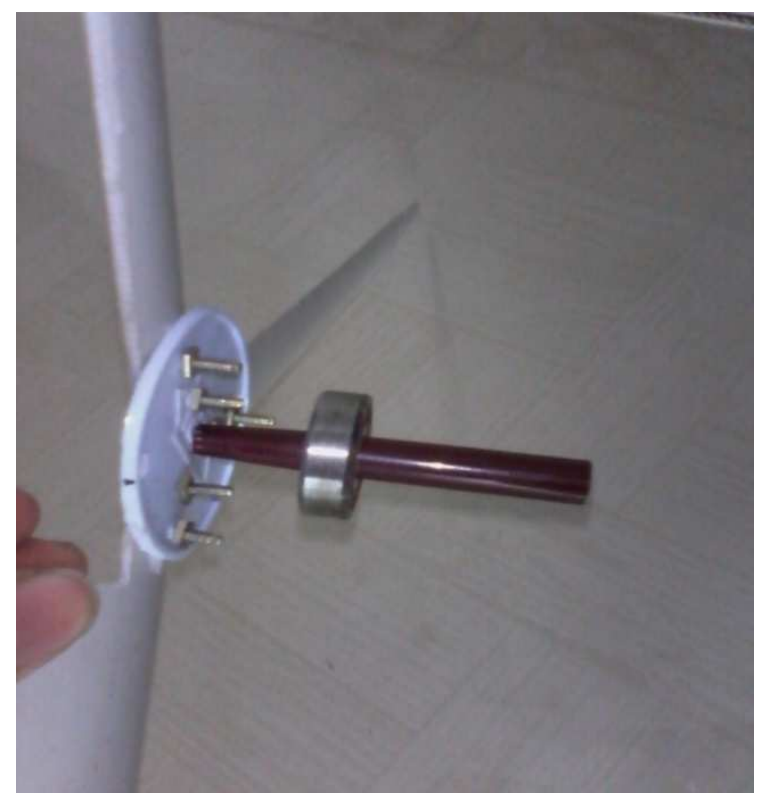

\section{Figure 6: Side View Showing Shaft and Bearing Fixed to Rotor}

Since the entire setup is small, obviously its size must be increased for practical application as poorly sized rotors result in an increase in cost than the revenue generated by the increased capacity factor. Increasing the size of the turbine rotors creates new challenges for manufacturers because, once the rotor size is designed as large as desired, the next thing is to get the blade efficiencies, increased which means study of fluid dynamic efficiency across a range of wind speeds."

\section{DIFFERENTIAL SET-UP}

The outputs of the two rotors are combined by using the concept of differential in internal combustion engines. The working principal of the differential is explained below.

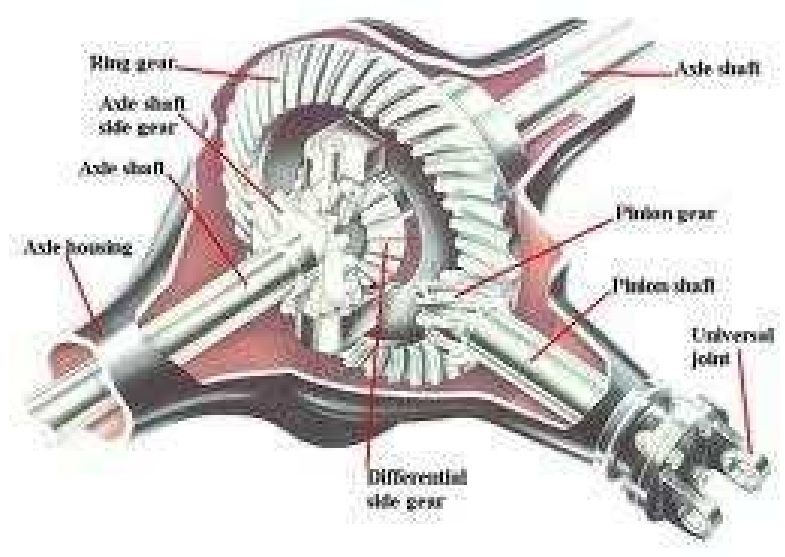

Figure 7: Cut Sectional View of Differential

Power from the engine is transmitted to ring gear through the pinion. The ring gear is connected to spider gear, which is at the heart of the differential. The spider gear is capable of two kinds of rotation first one is on the ring gear (rotation) and second on its own axis (spin). It is meshed with two side gears. When travelling straight, the spider gears do not spin on its axis, but rotates along with the ring gear as it meshes with the side gears it makes both the side gears rotate at the same speed. When the vehicle takes a turn on the right side the distance covered by the left tire is more. During this 
situation the peripheral velocities at the left side is the sum of rotational and spin velocities but on the right side it is the difference between the two. Therefore, the left wheel travels a larger distance than the right wheel. Similarly, when the vehicle has to take a left turn, the spider gear rotates in the opposite direction. During this situation, the peripheral velocities at the right side is the sum of rotational and spin velocities but on the left side it is the difference between the two. Therefore the right wheel travels a larger distance than the left wheel.

The same concept is used in combining the outputs of the two blades, the only difference being in the attaching the differential set-up to the rotor output. The side gears will be attached to the output of the blades and these side gears will mesh with the spider gear, which is attached to the ring gear. From the ring gear the rotation is transmitted to the turbine through the pinion gear. The ring gear size would be generally bigger as compared to the original set-up of the differential since this will increase the speed of rotation as the pinion size is smaller. The placement of the spider gear must be carefully worked, as the stress levels at the joints will be more. With the differential set-up for combining the output of the two rotors, there are issues or questions that need to be addressed. Firstly, when one is rotating at greater speed than the other, the differential will try to maintain the same speed by spinning and rotating action of the blade that has greater speed. This ultimately reduces the conversion rate of the high speed blade. The solution to this problem is increasing the contact area of the second rotor set-up for the bypassed air such that, the speed difference between the two rotor setups is minimized. This was tested and it was found that both the set-up had almost the same speed. In such a case, the torque that is transmitted to the turbine would be double. For the conversion rate to be improved, the size of the ring gear can be increased so that, the pinion which is coupled to the shaft of the turbine rotates at greater speed and the number of revolutions per revolution of the ring gear is high. Gearbox reliability is something which needs to be focused on.

\section{CONCLUSIONS}

It is seen that in conventional wind turbines, there is a loss of 50\% of air flow and with this set-up the amount of air that bypasses it is considerably reduced. The rotors are placed just behind the other and the outputs of these two rotors set-up are coupled, thereby reducing the conversion losses and mechanical losses. Thus, there is an increase in the conversion rate of air flow into useful power. Also, space requirements, installation costs and maintenance costs are considerably reduced.

\section{SCOPE}

The scope of this work for practical use includes optimization on

- Distance between front and rear rotors.

- Dimensions calculation in blade design for same speed for front rotor for rear end.

- Design of differential for this set- up.

- Stronger materials for column for preventing twisting action due to motion of two rotors.

- Materials for blades based on strength, weight, load carrying capacity.

\section{REFERENCES}

1. Anand Roa and dr. Shri Ram "Future and scope of wind energy in India" gjesr review paper vol. 1, issue 11, December, 2014

2. Joseph P. Hennessey jr "Some aspects of wind power statistics", Journal of applied metrology" Vol. 16 No.2 